\title{
Highly Durable and Flexible Paper Electrode with Dual Fiber Matrix Structure for High Performance Supercapacitors
}

Xiao Wu, ${ }^{1}$ Mingquan Zhang, ${ }^{1}$ Tao Song, ${ }^{1}$ Hongyan Mou, ${ }^{1}$ Zhouyang Xiang, ${ }^{1, *}$ Haisong Qi ${ }^{1,2}$

${ }^{1}$ State Key Laboratory of Pulp and Paper Engineering, South China University of Technology, Guangzhou 510640, China

${ }^{2}$ Guangdong Engineering Research Center for Green Fine Chemicals, Guangzhou 510640, China

*Corresponding author: Email: fezyxiang@ scut.edu.cn; Tel: +86-20-87113753

\section{Supplementary Figures}

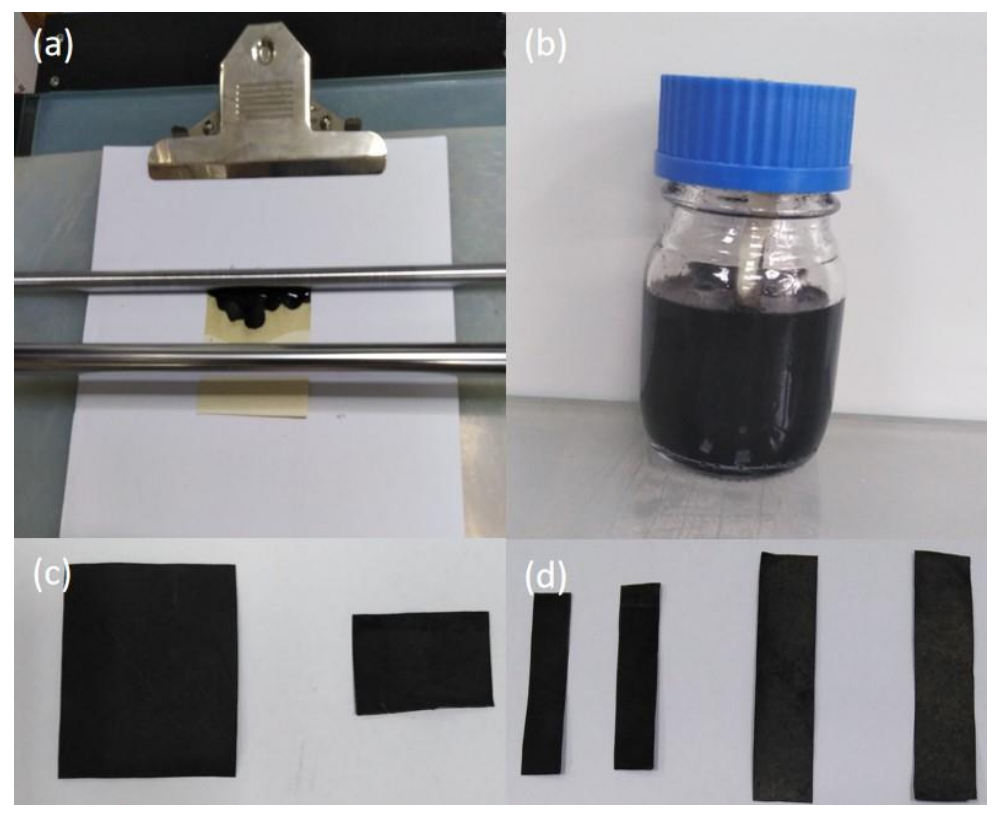

Figure S1. (a) Preparation of conductive paper by coating method using automatic coating machine; (b) preparation of conductive paper by soaking method; (c) the conductive paper prepared by coating method; (d) the conductive paper prepared by soaking method. 


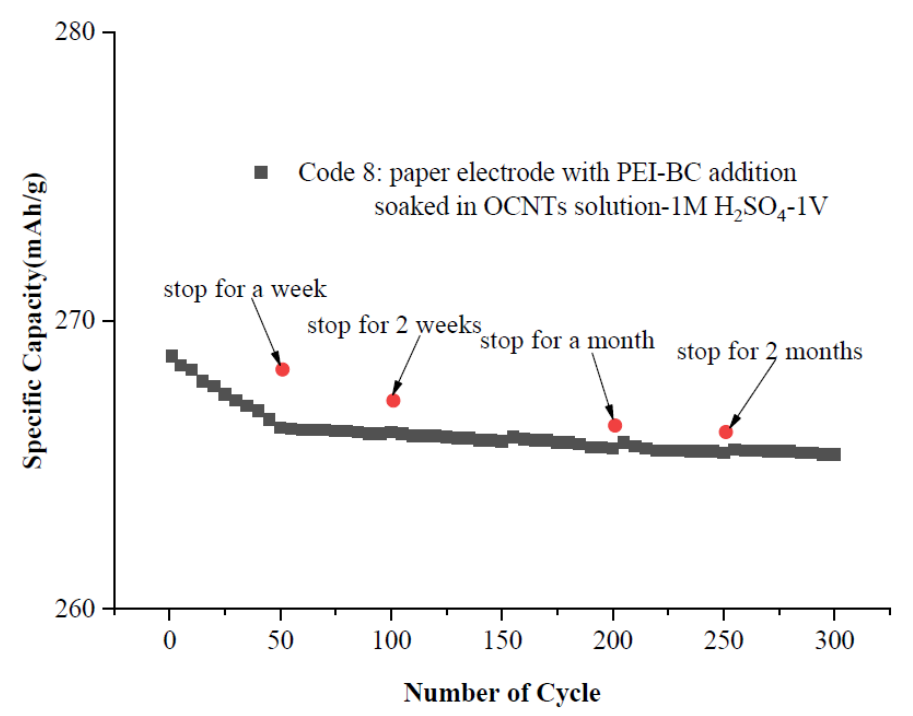

Figure S2. Specific capacity for paper electrode (code 8) measured at 300 charge/discharge cycles within a 2-month period and when the paper electrode stopped at different times in sulfuric acid electrolyte (the current density for the first 50 cycles was $8 \mathrm{~A} / \mathrm{g}$ and for the remaining 250 cycle was $6 \mathrm{~A} / \mathrm{g})$.

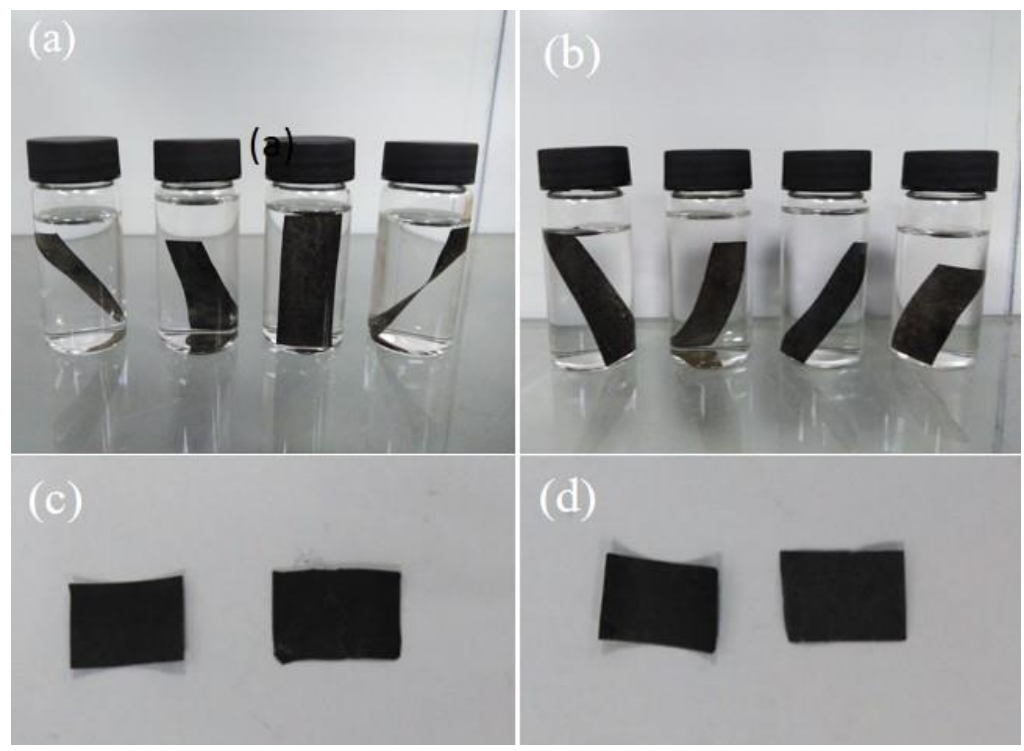

Figure S3. Tests on the stability of paper in the electrolyte: (a) The conductive paper was just soaked in sulfuric acid solution and organic electrolyte; (b) The conductive paper soaked in sulfuric acid solution for 2 months at room temperature (from left to right: code 7 paper in $1 \mathrm{M}$ sulfuric acid solution, code 8 paper in $1 \mathrm{M}$ sulfuric acid solution, code 7 paper in $0.25 \mathrm{M}$ organic electrolyte, code 8 paper in $0.25 \mathrm{M}$ inorganic electrolyte); (c) unprocessed conductive paper and (d) the conductive paper soaked in sodium sulfate solution for 2 months at room temperature (from left to right: code 7 paper in $1 \mathrm{M}$ sodium sulfate solution, code 8 paper in $1 \mathrm{M}$ sodium sulfate solution) 